Meta

Journal des traducteurs

Translators' Journal

\title{
Le degré zéro de la psychanalyse
}

\section{Pierre Ickowicz}

Volume 27, numéro 1, mars 1982

Psychanalyse et traduction

URI : https://id.erudit.org/iderudit/003162ar

DOI : https://doi.org/10.7202/003162ar

Aller au sommaire du numéro

Éditeur(s)

Les Presses de l'Université de Montréal

ISSN

0026-0452 (imprimé)

1492-1421 (numérique)

Découvrir la revue

Citer cet article

Ickowicz, P. (1982). Le degré zéro de la psychanalyse. Meta, 27(1), 113-118.

https://doi.org/10.7202/003162ar

Ce document est protégé par la loi sur le droit d'auteur. L'utilisation des services d'Érudit (y compris la reproduction) est assujettie à sa politique d'utilisation que vous pouvez consulter en ligne.

https://apropos.erudit.org/fr/usagers/politique-dutilisation/
Cet article est diffusé et préservé par Érudit.

Érudit est un consortium interuniversitaire sans but lucratif composé de l'Université de Montréal, l'Université Laval et l'Université du Québec à Montréal. Il a pour mission la promotion et la valorisation de la recherche. https://www.erudit.org/fr/ 


\section{LE DEGRÉ ZÉRO \\ DE LA PSYCHANALYSE}

PieRre ICKOWICZ

Ikh bin tristus

A zoï vi a vache à roulettes

Far vouss?

Far Gott ist nicht main calé

Ikh will nicht zaïn a kalike

Fin dai Leiben

Ikh will bloïss blaben in de eretz Je veux tout simplement

Makhen a rodeo mit dai raidera Mettre mes pas dans mes pas

Nicht gaïn foyeré avek

Wen de epis andech

Wet se spatchieren mit

Zannen froukhten

Zan steppes

Zanen feyets

Yo! Gott is nicht main calé

Eye existiyet

Zikhe! Zikhè!

Sot mai guikhoulim

A shabbat mit in die Vokh

Mitwokh a vort

hot me guezougt

Gait! Gait! Gaiit

$\mathrm{Vi}$ ?

Knob ze gue fraigt

Gait! Gait! Gait

Hot me gue entfert

Oh! la la

Rekht links

En haut, en bas

Gaït! Gaït! Gaït zougt zé

Sis me nicht guit

Ikh must gain

Ikh gaïn shoyn

Gaïn, gaïe nicht avek unt miye And alle maïne menschen
Quelle est cette tristesse qui m'imbibe

Quand je pense aux vaches espagnoles?

Quel est ce pourquoi?

C'est que Dieu ma fiancée n'est pas.

Je ne me résouds pas à être

un clopin-clopant de la vie.

A la surface de la terre

Faire gambader le dire au mouvement des mots

Et ne pas m'en aller

Avant que ce petit quelque chose

Aille se promener, attelé

À ses fruits

Ses steppes

Et ses chevaux du désert.

C'est bien ça, Dieu ma fiancée n'est pas

C'est de la certitude, c'est indoutable.

Un rêve s'est rêvé en moi

Un de ces jours de fête qui n'attend pas le calendrier

Un de ces mercredis, un dit m'a dit :

Va, va, va

Où donc?

Ai-je lancé en écho

Va, va, va

Ai-je entendu encore

Où? Oh! Ah!

À l'Est ou à l'Ouest?

Au zénith ou à l'intérieur de la terre?

Va, va, va dit le dit

Quelle est cette fatigue sidérale?

Il me faut aller

Je suis au bord de l'exode

Départ ne part pas sans m'emmener. 
Une fable empruntée au film de R. Polanski le Bal des Vampires peut me servir de balise et de valise à constituer l'exil de l'enfance en exode de l'écrire à la découverte évidente et immaîtrisable d'un écart.

Le professeur qui étudie les vampires se trouve convié à un de leurs bals. Le propre d'un vampire c'est de manquer de reflet; le manque de reflet reflète en négatif l'espace possible d'un manque. Au cours de ce bal le professeur danse un menuet avec une vampire, ce qui l'amène devant un miroir où son image apparaît. Apparition qui déchire le voile de l'illusion et brise la danse. Son image se forme en ce lieu où l'autre se voit ne se voyant pas. Cette rencontre entre l'apparition de l'image de soi et la découverte qu'il y a un autre, au moment précis où la mère découvre que son enfant ne la satisfait plus tandis que l'enfant appréhende qu'elle ne l'a jamais satisfait, quand ce qui relie l'un à l'autre efface ce qui les unit, on est à un degré zéro.

C'est ce moment fictif que je vais m'efforcer de formuler: l'écart de la langue au discours.

Franz Kafka s'est désigné une fois comme un «invité de la langue allemande». Ce même Kafka qui a tenu en allemand une conférence: «Discours sur la langue yiddish » face à des auditeurs supposés ne pas le parler, l'écoutant lui, censé ne pas le connaître. Freud, quant à lui, dès l'adolescence, est perçu par un de ses professeurs, qui reprendrait une expression de Johann Gottfried Herder, comme ayant un style «idiotique» c'est-à-dire, comme Freud l'écrit à Emil Fluss, «un style qui est à la fois correct et caractéristique».

Freud se sentait l'invité du désir et le commensal de la langue allemande. Cette langue allemande, il l'invoque pour justifier l'opposition instinct sexuel/instinct de conservation (dans «Pulsion et destin des Pulsions»); il l'invoque encore pour justifier le lapsus et l'oubli dans Introduction à la Psychanalyse; et il l'utilise subtilement en écrivant dans son texte sur la Dénégation un «made in Germany», y mêlant de l'anglais et la trace du lieu de l'emprunt.

Cette langue, il la fait parler à son insu, insu qu'il witzise du côté du passage du rêve au texte et du côté des moyens que se donne le discours pour se maintenir tendancieux (le Mot d'esprit et ses rapports avec l'inconscient).

Cet insu de la langue allemande, en tant qu'il s'est noué à un in-dit de Freud, a donné l'inconscient. C'est à cette place que nous situerons un rapport de Freud à la langue yiddishe. Langue yiddishe qu'il ne peut s'empêcher de citer: «Azoï!» «Oï Poï Vaï», «bubi-zin, Nem-Rod» et dont il mimera en toute naïveté le mimétisme.

Cette hypothèse est une construction; notre ignorance relative du yiddish et le fait que Freud ait laissé la trace de ce qui l'a dérangé nous y a rendu sensible sous sa plume.

Cette construction se situe sur le versant de la résistance de censure, entre la révélation et la formulation; esquisse pour établir le texte.

Établir le texte, c'est rencontrer ce qu'il dit, l'entendre pour percevoir la lacune qui le constitue. Or, si Freud est considéré comme un médecin, un spécialiste des maladies nerveuses, un esprit curieux, un lettré qui avait l'originalité de s'intéresser à des questions pour lesquelles il n'avait pas été habilité, 
ce qu'établit l'hypothèse du yiddish, c'est que la constitution d'un discours sur l'inconscient est corrélative de la survenue du dire du yiddish dans son discours.

Si l'expérience d'une langue rattache une génération à une autre génération, elle relie les géniteurs entre eux dans le contexte des conditions d'exploration collective de la langue.

La tendance à instrumentaliser le rapport à la langue tant comme apprentissage du langage que comme langue-culture soutenue par des ceuvres séparées des parleurs est démontée par la langue yiddish qui porte dans son développement la nécessité de son exploration.

Le parleur est le véhicule de l'intention que le yiddish transporte.

Le parler est l'actualisation de l'interprétation qui inaugure le yiddish.

La rencontre est la mise en commun d'un inoubli que le fait de parler maintient.

On peut supposer que la présence du yiddish dans le mouvement de l'écriture de Freud repose sur un choix de Freud (qui se présente comme la Terre promise de cette langue) et sur un effet du yiddish (qui confronte Freud à l'exode) et occupe alors par rapport à cette écriture la place de l'interprète. Si le yiddish interprète ses emprunts, Freud lui emprunte sa position interprétative.

Le yiddish se dit à travers Freud qui l'écrit. Freud apporte le yiddish comme un emprunt qui donne vie au texte et reste fragment vivant du texte. Fragment ombilic. Reste qui porte la totalité. Signe de la transmission. Dire se nommant.

Freud réalise ainsi deux souhaits: acheminer la tradition que le yiddish rend sensible comme transmission, écrire la relation au monde du point de vue de l'objectivité.

Le yiddish dit dans l'écriture de Freud ce que cette écriture écrit; cela nous amène à cette question : la psychanalyse transmet-elle le yiddish? En quoi si elle s'en est inspirée, ce qui l'inaugure prolonge-t-il ce qui inaugure le yiddish ? Ou encore : la psychanalyse est-elle un discours sur la langue yiddish? L'acheminement méconnu de ce qu'elle accomplit?

Pour reprendre une allégorie métaphorisée de Freud, on pourrait proposer ceci : la langue yiddish et le discours de Freud seraient les deux armées qui garderaient la frontière, qu'il identifie au Moi dans Inhibition, symptôme et angoisse, à la différence, essentielle, que ces deux armées ne sont pas ennemies; elles ne sont pas davantage amies et ne gardent pas la frontière mais l'occupent. L'une est la trace d'un mouvement interne, vers la frontière, l'autre la limite de ce qui a été emprunté au monde extérieur sans vraiment pouvoir franchir cette frontière. Le discours de Freud invente (à la frontière) la frontière du yiddish, ce qui lui permet de rencontrer les limites et de les déplacer, déplacement vers cette frontière du yiddish.

Si les Trois essais sur la théorie de la sexualité traduisent la rupture de Freud avec le mode de pensée médico-légal, le yiddish intercalé rend sensible que l'horizon de Freud était le lointain du yiddish et non l'ambition d'être le pape de la psychiatrie et le grand ordonnateur de la psychopathologie.

Le lointain du yiddish est le lointain de n'importe quel parler quand il découvre sa frontière à la formation des mots et à l'effet de structure qu'elle 
transporte pour véhiculer des intentions. Il se fait que le yiddish est le parler d'un groupe minoritaire qui excursionne dans l'élaboration du groupe majoritaire, ce qui le met dans une position différente, non de nature, mais de situation, d'un patois qui croit être lié à une zone de parler et à une frontière géographique d'intention.

Celui qui quitte sa région peut garder un accent et abandonner son parler. Le parler de la ville annulerait le patois.

Celui qui quitte le groupe minoritaire est confronté à une identification partagée par l'exploration de deux parlers. Le yiddish ayant une double identité : l'alphabet de l'hébreu et l'emprunt sonore de son inauguration, l'allemand, anticipe sur cette difficulté; il résiste à l'annulation en empruntant au parler qui pourrait le supplanter et en l'écrivant autrement.

Ce fait installe le yiddish à la frontière. Là où on s'attend à trouver une langue nationale, on découvre la vie des mots. Là où on suppose une langue dominée que l'on historicise et un progrès que l'on géographise, on rencontre l'inoubli, une emprise active sur l'actuel, et une incompatibilité entre une langue imposée et le fait de parler. Symptôme de la langue, il donne corps au parleur dans le rapport à une autre langue.

L'hypothèse du yiddish vise à établir le texte comme effet de l'effacement de la trace et accueil du non-effacé.

Plus nous nous sommes attachés à montrer que la présence du yiddish dans l'écriture de Freud devait dépendre d'une expérience directe du yiddish - son père le parlait-il? sa mère le parlait-elle? d'où venait donc la présence du yiddish? - plus nous nous sommes rendus compte que la question ne se réduisait pas à établir qu'il l'avait entendu mais à formuler en quoi le yiddish était la trace d'un entendu. D'autant plus qu'il semble que Freud appartenait à une zone géo-historique où on avait jeté l'excommunication sur le yiddish en utilisant l'alphabet hébreu pour y écrire de l'allemand moderne, et effacer les divers emprunts conservés par le yiddish. Il était important de déterminer comment il avait accueilli la trace de cet entendu, surtout s'il ne l'avait pas parlé et la place qu'il lui faisait occuper dans l'organisation de son discours.

Freud n'a pas accueilli le yiddish en «juif ». Le juif qui est désigné dans le mouvement d'un langage d'insulte ne parle pas le yiddish, quand il le parle, ce n'est pas un juif, c'est un parleur. Il l'a accueilli comme un héritage d'intentions. La rupture avec la langue coïncide toujours avec un dérèglement identificatoire que le délire pérennise en essayant d'inventer une langue de passage où règne le signifiant comme jeu de l'inoubli.

La présence de cette langue, au-delà de cette rupture, sa survenue au rêve construit un passage qui justifie à la fois que le yiddish est une langue mnésique pour Freud et que la préhistoire de la psychanalyse, l'amnésie infantile de Freud, doit se soutenir d'une présence de cette langue.

Le yiddish apparaît dans la situation d'un refoulé originaire. L'écrire de Freud traitunairise le dire discontinu du yiddish à l'écrit.

La rencontre avec ce refoulé originaire met en jeu le «refoulement proprement dit» en faisant intervenir la langue allemande. Refoulé originaire et «refou- 
lement proprement dit» cohabitent dans une synchronie signifiante. Le Mot d'esprit dans ses rapports avec l'inconscient est la mise en forme des techniques qui lui donnent une orientation tendancieuse. Le fait que Freud ait modifié l'allemand moderne et que le yiddish ait conservé de l'allemand ancien a permis cette rencontre de deux états de la langue et de deux conjonctures historiques chronologiquement séparés.

Là où le yiddish se cherche un avenir à un horizon de sa frontière, l'allemand de Freud fait apparaître le yiddish comme le passé vivant de l'allemand. La psychanalyse dilue le passage de l'allemand ancien à l'allemand moderne, ce qui permet au refoulement de révéler un refoulé originaire. La déformation qu'il impose Freud à la langue allemande dessine l'ombre du yiddish, yiddish qui a maintenu sa propre progression et rendu sensible une autre progression que celle de l'allemand. Freud a permis cette rencontre qui a laissé le yiddish et le texte de Freud séparés, reliés par l'écriture. Le yiddish il ne le traduit pas, il l'expose; en interrogeant le non-effacé de la trace, il a rencontré le non-effacé du yiddish.

La psychanalyse serait la traduction du yiddish avec les moyens du yiddish; un yiddish scientifique qui aurait atteint sa Terre promise mais qui resterait du yiddish en conservant les caractéristiques qui inaugurent le yiddish.

Le yiddish traduit, a un billet aller, il n'a pas de retour, le yiddish fournit le billet aller, Freud fournit le billet de retour.

La présence du yiddish est un aveu non camouflé, le contraire de ce qui est caviardé : le yiddish existe, sous-entendu "Moi aussi Freud j'existe» et c'est en même temps une interrogation: - $\mathrm{Si}$, alors que le yiddish existe, on ne prend pas la mesure de sa réalité, en quoi moi Freud qui existe prendra-t-on plus compte de ma réalité? Traitez-moi au moins comme je traite le yiddish. Appel auquel Lacan, pourrait-on dire, a répondu à sa manière.

Arrêtons-nous.

En quoi dire qu'une chose existe est un aveu? En quoi l'aveu est-il le détour nécessaire à l'affirmation de l'existence? Le déguisement qui nous vient du rêve doit-il s'accomplir comme dissimulation des intentions, en liant l'acte d'énonciation au fait de dire ce qu'elle ne dit pas? La langue yiddish permet d'inverser la dissimulation et d'en faire une révélation. La cohabitation du yiddish et du texte, le mouvement de l'écriture de Freud, nous rend sensible l'irruption de cet espace qui a fait événement pour lui dans l'écart du noneffacé et du non-effacement.

Avançons vers une conclusion en laissant travailler cette histoire évoquée par Freud dans le Mot d'esprit.

Le médecin qui doit assister à l'accouchement de la baronne déclare que le moment n'est pas encore venu et propose au baron une partie de cartes dans la chambre voisine. Quelque temps après, un appel de la baronne en français retentit à l'oreille des deux messieurs : «Ah! mon Dieu que je souffre !» Le mari sursaute mais le médecin reste calme : «Ce n'est rien, jouons toujours.» 
Un peu plus tard un gémissement, cette fois en allemand: «Dieu! Dieu! que je souffre!»

"Voulez-vous entrer, Monsieur le professeur? dit le baron.

- Ce n'est pas encore le moment.»

Enfin, on entend dans la chambre voisine un cri inarticulé en yiddish : «Oï, Oï, Vaii! » Alors le médecin jette les cartes et dit : «C'est le moment.»

Cette histoire vient révéler comment les langues s'écartent à mesure que la vérité se fait plus proche pour laisser parler les plus oubliées. Au moment ultime où la mère réalise cet autre qu'est l'enfant qui naît, elle laisse échapper le cri du yiddish. Premier signifiant de rencontre avec le langage pour le nouveauné. Le Professeur qui, comme Freud, aime jouer aux cartes ne s'y trompe pas, attentif à l'entendu du premier signifiant recouvert par la sédimentation des langues.

Le refoulé qui se constitue du recours à une langue rend sensible le refoulement dans une autre langue.

Le français d'abord qui a permis à Freud d'intercaler la formule «nonarrivé» qu'il emploie depuis les Psychonévroses de défense jusqu'à Un trouble de mémoire sur l'Acropole; la reconnaissance de ce qui s'effectue se produit comme «non-arrivé». "Non-arrivé» dit le français à Freud. «Non-arrivé», que reprend l'allemand de La dénégation pour y dessiner le corps de la mère. «Ö̈ $V a i ̈ »$ enfin qui se soutient d'un sous-entendu : «Miye nebekh». Le cri du yiddish qui traverse la gorge de la mère troue l'imaginaire de l'emprunt, laissant en pointillé à ce dont elle accouche le soin de poursuivre. "Oï Vaï! Fils de ma peine. Miye Nebekh. C'est bien de moi qu'il s'agit quand ça crie.»

Si comme le roi Boabdil que cite Freud dans Un trouble de mémoire sur l'Acropole qui apprenant la perte d'Alhama et du même coup la fin de son règne, brûle le texte qui l'écrit et tue le messager qui le porte.

Cartas le fueron venidas

De que Alhama era ganada

Las cartas echo en el fuego

$\mathrm{Y}$ al mensagero mataba.

Freud a lui-même détruit ses premiers textes en les brûlant; il en diffère en ce que son ambition le constitue en messager d'un message dont le cri qui l'annonce laisse en pointillé les mots qui le prolongent.

L'insignifiance du pré-analytique n'est-ce pas ce fragment silencieux qui est l'écho d'un cri? Cette histoire toujours reprise jusqu'à ce que Freud parvienne à l'insérer dans un discours?

Le corpus de l'œuvre freudienne ne se constitue-t-il pas à ce moment qui inaugure ce qui, en séparant Freud du corps de la mère l'introduit aux mots qui portent le désir, en yiddish dans le texte?

Le yiddish ne cessera de brûler, brasero d'un discours.

Il est temps pour moi, de reparcourir le silence en silence, vous laissant attentifs au silence du pré-analytique. 